\title{
Mental Pain, Psychological Distress, and Suicidal Ideation During the COVID-19 Emergency: the Moderating Role of Tolerance for Mental Pain
}

\author{
Giulia Landi ${ }^{1,2}$ (D) Ariella Grossman-Giron ${ }^{3,4}$ - Dana Tzur Bitan ${ }^{3,4} \cdot$ Mario Mikulincer $^{5}$. \\ Silvana Grandi ${ }^{1,2}$. Eliana Tossani ${ }^{1,2}$
}

Accepted: 8 September 2021 / Published online: 1 November 2021

(c) The Author(s), under exclusive licence to Springer Science+Business Media, LLC, part of Springer Nature 2021

\begin{abstract}
The psychosocial stressors related to the ongoing COVID-19 pandemic and associated lockdowns have been shown to lead to an exacerbation of suicide risk. The present study aims to examine (a) the contribution of mental pain intensity to psychological distress and suicidal ideation during the COVID-19 pandemic and (b) the protective role of mental pain tolerance in buffering these adverse mental health effects. A total of 652 adults $(74.2 \%$ female, $M=33.99$ years, $S D=13.74$ ) were assessed through an online survey during the first mandatory lockdown in Italy. Participants completed measures of mental pain intensity and tolerance, psychological distress, and suicidal ideation. Results showed that mental pain intensity significantly predicted increases in psychological distress and suicidal ideation while mental pain tolerance significantly buffered the adverse effects of mental pain intensity on psychological distress and suicidal ideation. The findings highlight that tolerance for mental pain may act as a powerful protective factor during the pandemic. Evidence-based public health interventions fostering tolerance for mental pain during a pandemic are needed in order to effectively reduce suicide in potential risk groups.
\end{abstract}

Keywords COVID-19 pandemic $\cdot$ Mental pain · Tolerance for mental pain · Psychological distress $\cdot$ Suicidal ideation

The Coronavirus disease 2019 (COVID-19) outbreak worldwide has led many countries to issue unprecedented physical distancing measures in an attempt to contain the spread of the

Giulia Landi

giulia.landi7@unibo.it

1 Department of Psychology, University of Bologna, Viale Berti Pichat 5, 40127 Bologna, Italy

2 Laboratory of Psychosomatics and Clinimetrics, Department of Psychology, University of Bologna, Viale Europa 115, 47023 Cesena, Italy

3 Department of Behavioral Sciences, Ariel University, Ariel, Israel

4 Shalvata Mental Health Center, Hod Hasharon, Sackler School of Medicine, Tel Aviv University, Ramat Aviv, Israel

5 Interdisciplinary Center Herzlyia, P. O. Box 167, 46150 Herzliya, Israel 
virus and to alleviate the burden on healthcare systems. Many countries, severely affected by the pandemic, employed national lockdowns, which included quarantines, reduced social contacts, and limitations on movements of the general populations. A large body of evidence indicates that these restrictions, accompanied by the intense fear of severe illness, loneliness, financial problems, and uncertainty over the course of the pandemic, have profound adverse mental health implications (Bakioğlu et al., 2020; Besser et al., 2020; Brooks et al., 2020; Daly \& Robinson, 2021; Landi et al., 2020b; Pakenham et al., 2020). Meta-analyses of community-based studies revealed a pooled prevalence of $18-33 \%$ of depressive symptoms (Bueno-Notivol et al., 2020) and 24-31\% of anxiety symptoms (Kan et al., 2021) during the first months of the COVID-19 emergency. A systematic review, Xiong and colleagues (2020) further reported an increase in rates of psychological distress following the emergence of the pandemic among the general population in countries such as China, Spain, Italy, the USA, Turkey, Nepal, and Denmark (Xiong et al., 2020).

\section{Suicidal Ideation During the COVID-19 Pandemic}

The adverse psychological outcomes and the psychosocial stressors related to the COVID19 crisis have led researchers worldwide to raise concerns regarding the potential raise of suicidal behaviors during and after the pandemic (Gunnell et al., 2020; Leaune et al., 2020; Mamun et al., 2020). For example, an online study carried out in the UK on a national representative sample of 44,775 people revealed that $18 \%$ of participants reported experiencing suicidal thoughts in the first month of lockdown, whereas $5 \%$ of participants reported harming themselves at least once since the start of lockdown (Iob et al., 2020). Accordingly, another online survey conducted in the USA indicated that $17.5 \%$ of respondents reported suicide ideation, whereas $4.9 \%$ had attempted suicide since the beginning of the COVID-19 pandemic (Ammerman et al., 2021). Finally, a recent systematic review and meta-analysis of 54 studies $(N=308,596)$ revealed an increase in rates of suicidal thoughts (10.81\%), suicide attempts (4.68\%), and self-harm (9.63\%) during the COVID-19 pandemic relative to rates documented in pre-pandemic investigations (Dubé et al., 2021).

These disconcerting trends have led researchers to identify relevant risk and protective factors for suicidal ideation and psychological distress during the COVID-19 pandemic in order to aid decision-makers and clinicians to develop appropriate interventions (Niederkrotenthaler et al., 2020a, b). For this reason, the current study explored the role of mental pain as a risk factor for suicidal ideation and psychological distress, and of tolerance for mental pain, as a protective factor during the first lockdown of the COVID-19 pandemic (e.g., Becker et al., 2019; Orbach et al., 2003).

\section{Mental Pain Intensity and Tolerance as Potential Risk and Protective Factors During the COVID-19 Pandemic}

Mental pain, also known as "psychache" (Shneidman, 1993), is defined as an acute state of intense psychological pain associated with negative cognitive and emotional aspects of the self-i.e., thoughts of self-disappointment or inadequacies and feelings of guilt, anguish, fear, panic, angst, loneliness, and helplessness often accompanied by a sense of disconnection, loss, or incompleteness of the self (Bolger, 1999; Orbach et al., 2003; Tossani, 2013). Mental pain is viewed as stemming from the discrepancy between the ideal and the 
actual perception of self, accompanied by the awareness of one's role in the experience of emotional pain (Tossani, 2013). Over the years, a growing body of evidence has underlined the transdiagnostic nature of this construct as mental pain has been found to be a risk factor for a wide variety of various psychiatric problems, ultimately regarded as one of the most important proximal predictors of suicidal risk well beyond depression (e.g., Montemarano et al., 2018; Tossani, 2013; Verrocchio et al., 2016).

Conversely, tolerance for mental pain, or the extent to which one can endure and manage the aversive state of mental pain, has been considered a protective mental health factor related to adaptive responses to psychological pain during stressful periods (Meerwijk et al., 2019). As such, tolerance for mental pain has been associated with higher emotion regulation skills (e.g., Becker et al., 2019) and with lowered depressive symptomatology and suicidal ideation (e.g., Landi et al., 2020a, b; Meerwijk \& Weiss, 2018). Moreover, the level of tolerance for mental pain has been shown to differentiate suicidal attempters from non-suicidal psychiatric inpatients (e.g., Levinger et al., 2015; Segal-Engelchin et. al, 2015; Shelef et al., 2015). Thus, whereas the intensity of mental pain seems to be associated with heightened psychological distress and suicidality, findings suggest that mental pain tolerance seems to function as a resilience-fostering, protective factor. These mental-health repercussions of mental pain intensity and tolerance may be especially relevant during the COVID-19 pandemic and lockdowns as individuals have been found to report elevated levels of psychological distress and suicidal ideations.

\section{Study Aims}

This study aimed to examine the role of mental pain intensity and tolerance in predicting psychological distress and suicidal ideations during the first COVID-19 lockdown in Italy. The research hypotheses were as follows: (a) mental pain intensity would act as a risk factor for negative mental health indicators, so that the higher the reported intensity of mental pain, the more severe the psychological distress and suicidal ideation during the lockdown; (b) tolerance for mental pain would act as a moderating factor, buffering the adverse effects of intensity of mental pain on psychological distress during the lockdown. Furthermore, we aimed to examine the protective role of mental pain tolerance with regard to suicidal ideation. We hypothesized that (c) tolerance for mental pain would weaken the link between mental pain intensity and suicidal ideation during the pandemic lockdown.

\section{Methods}

\section{Participants and Procedure}

This cross-sectional survey took place during the first Italian mandatory lockdown between April and May 2020. Inclusion criteria were living in Italy and being a minimum of 18 years old. Recruitment was conducted through social media. The survey was advertised as a research designed to investigate the psychological effect of the COVID-19 emergency, and was administered online and took approximately 15-20 min to complete. The study was approved by a university institutional human research ethics committee and participants signed an online informed consent. 


\section{Measures}

\section{Demographics and COVID-19 and Lockdown-related Variables}

Participants indicated their age, gender, marital status, education, employment, and SES. The following lockdown-related information was also collected: the number of days in lockdown, testing positive for COVID-19, and family members testing positive or deceased due to COVID-19.

\section{Intensity and Tolerance for Mental Pain}

The intensity of mental pain was assessed with a 31-item Italian version of the Orbach \& Mikulincer Mental Pain scale (OMMP; Orbach et al., 2003), translated and validated by Tossani et al. (2021). This scale taps the extent to which mental pain is perceived as irreversible (e.g., "The difficult situation will never change") and the extent to which people experience pain-related narcissistic wounds (e.g., "I feel abandoned and lonely"), emotional flooding (e.g., "My feelings change all the time"), freezing and loss of control (e.g., "I feel paralyzed"), and feelings of meaningless and emptiness (e.g., "I can't find meaning in my life"). Participants rated each item on a 5-point scale, ranging from 1 (not at all) to 5 (very strongly). The Italian version demonstrated good psychometric properties (Tossani et al., 2021; $\alpha=0.89$ in the current sample).

The Italian version of the Tolerance for Mental Scale-10 (TMPS-10; Meerwijk et al., 2019), translated and validated by Landi, Furlani, and colleagues (2020a) was used to assess the extent to which people can manage the pain (e.g., "I can get the pain out of my mind,") or endure it (e.g. "I believe that the time will make the pain disappear"). Items are rated on a 5-point scale, ranging from 1 (not true) to 5 (very true). The scale demonstrated good psychometric properties (Meerwijk et al., 2019; $\alpha=0.89$ in the current sample).

\section{Psychological Distress and Suicidal Ideation}

We operationalized psychological distress with the three outcomes: peritraumatic distress, anxiety, and depression. Specifically, we used the COVID-19 Peritraumatic Distress Index (CPDI; Qiu et al., 2020) to assess peritraumatic distress due to the COVID-19 emergency (e.g., "I cannot sleep well. I always dream about myself or my family being infected by COVID-19" or "I avoid watching COVID-19 news, since I am too scared to do so"). Items are rated on a 5-point scale $(0=$ never to $4=$ most of the time $)$. In a confirmatory factor analysis of the Italian version, Landi, Pakenham et al. (2020b) eliminated 5 items with low factor loadings and found a good one-factor model fit ( $\alpha=0.89$ in the current sample). Anxiety and depression were assessed with the Italian versions (Kroenke \& Spitzer, 2010) of the General Anxiety Disorder Scale (GAD-7; Spitzer et al., 2006) and of the Patient Health Questionnaire (PHQ-9; Spitzer et al., 1999). Items were rated on a 4-point Likert scale $(0=$ not at all to $3=$ nearly every day). These measures have been shown to be psychometrically sound in previous studies (Kroenke \& Spitzer, 2010; $\alpha=0.90$ and $\alpha=0.87$ in the current sample). In order to assess suicidal ideation, we isolated participants' responses to item 9 of the PHQ-9 (i.e., "Thoughts that you would be better off dead, or of hurting yourself”), which is a common screener for suicidal risk (e.g., Simon et al., 2013). 
Table 1 Descriptive data and correlations between mental pain intensity and tolerance, gender, age, psychological distress, and suicidal ideation

\begin{tabular}{llrlrrr}
\hline & $M(S D)$ & Range $\begin{array}{l}\text { COVID-19 } \\
\text { distress }\end{array}$ & Anxiety & Depression & Suicidal ideation \\
\hline $\begin{array}{l}\text { Mental pain } \\
\text { intensity }\end{array}$ & $2.36(0.79)$ & $1-4.71$ & $0.74 * * *$ & $0.71^{* * *}$ & $0.80^{* * *}$ & $0.46^{* * *}$ \\
$\begin{array}{l}\text { Tolerance for } \\
\text { mental pain }\end{array}$ & $3.84(0.65)$ & $1.80-5$ & $-0.42^{* * *}$ & $-0.41^{* * *}$ & $-0.45^{* * *}$ & $-0.39 * * *$ \\
$\begin{array}{l}\text { Gender a } \\
\text { Age }\end{array}$ & $73.9 \%$ & & $-0.29 * *$ & $-0.26^{* *}$ & $-0.25^{* *}$ & -0.06 \\
\hline
\end{tabular}

${ }^{\mathrm{a}}$ Gender $0=$ female, $1=$ male. Pearson correlations are displayed for continuous variables and Spearman correlations for gender

${ }^{*} p<0.05 ;{ }^{* *} p<0.01 ;{ }^{* * *} p<0.001$

\section{Statistical Analysis}

All analyses were performed in SPSS 24 and with Process v.3.5. Before conducting moderation analyses, we examined the skewness and kurtosis values of all study variables. The values were acceptable for all variables except for suicidal ideation, which was positively skewed (skew $=2.91, S D=0.96$ ). To correct for skewness, according to guidelines by Curran et al. (1996), we log-transformed suicidal ideation and skewness was checked again and found to be within acceptable limits ( test the moderating role of tolerance for mental pain (W variable) in the link of mental pain intensity (X variable, focal predictor) to psychological distress and suicidal ideation (Y, dependent variables), moderation analyses were performed with Process Model 1 (Hayes, 2018). Moderation was established when the interaction $(\mathrm{X} \times \mathrm{W})$ was significant (Hayes, 2018). As gender and age were the only demographics that significantly correlated with mental health outcomes, we controlled for them in moderation analyses (see Table 1). In order to reduce potential problems related to high multicollinearity, both the focal predictor (mental pain intensity) and the moderator (tolerance for mental pain) were mean-centered in all moderation analyses. To further probe the interaction, values of the moderator were selected (e.g., high $=+1$ $\mathrm{SD}$ above the mean, average $=$ mean, and low $=-1 \mathrm{SD}$ below the mean) and the effect of the focal predictor (mental pain intensity) at those values was estimated (Hayes, 2018).

\section{Results}

\section{Sample Characteristics}

The sample was composed of 652 adults, $75.3 \%$ female, aged 18 to $81 \quad(M=38.8$, $S D=13.2)$. Approximately half of the sample $(48.1 \%)$ had a bachelor's degree, and $26.4 \%$ completed high school. Almost half $(46.0 \%)$ of the sample were either married or living with a partner, while $7.4 \%$ were widowed or divorced. Most participants $(81.3 \%)$ indicated to belong to the middle socioeconomic band, $10.6 \%$ to the lower and $8.2 \%$ identified themselves as wealthier than the average. More than half of the participants $(66.4 \%)$ were employed, $11.2 \%$ were students, and $9.6 \%$ were unemployed. The mean days spent in 
lockdown was $35.70(S D=8.41)$. A total of 178 participants $(18.9 \%)$ reported having been infected by COVID-19. A quarter of the sample $(25.5 \%)$ reported having family members infected by COVID-19, 16.7\% died due to COVID-19. Participants exhibited a prevalence of $18-32 \%$ of moderate to severe psychological distress, ${ }^{1}$ with $15.3 \%$ of the sample displaying the presence of suicidal ideations $(10.1 \%$ several days, $2.5 \%$ more than half of the days, and $2.8 \%$ nearly every day).

\section{Links of Mental Pain Intensity to Psychological Distress and Suicidal Ideation During the COVID-19 Pandemic}

Our first prediction that mental pain intensity would be associated with higher psychological distress and suicidal ideation during the COVID-19 pandemic and mandatory lockdown was supported (see Table 1). Correlations of mental pain intensity with heightened psychological distress were significant and of a large magnitude, ranging between 0.71 and 0.80 , all $p s<0.001$. Furthermore, mental pain displayed a moderate to large significant association with heightened suicidal ideation, $r=0.46, p<0.001$.

\section{The Protecting Role of Tolerance for Mental Pain During the COVID-19 Pandemic}

To examine the prediction that tolerance for mental pain would buffer the association between mental pain intensity and increases in psychological distress, three simple moderation analyses were conducted. The interaction between mental pain intensity and tolerance for mental pain was significant for peritraumatic distress $(\beta=-0.043, S E=0.015$, $p<0.01)$, anxiety $(\beta=-0.023, S E=0.007, p<0.001)$, and depression $(\beta=-0.031$, $S E=0.006, p<0.001)$. As indicated in Table 2, the full models predicted between 54.1 and $66.6 \%$ of the variance in psychological distress, whereas the interaction exclusively predicted between 0.5 and $1.4 \%$ of the variance. Mental pain and all levels of tolerance for mental pain (low, average, and high) were significantly related to psychological distress. A further examination of the source of the significant interaction (see Fig. 1) revealed that higher tolerance for mental pain significantly weakened the positive link of mental pain intensity to psychological distress.

To analyze the prediction that tolerance for mental pain would buffer the association between mental pain intensity and heightened suicidal ideation, one simple moderation analysis was carried out. Results showed that the interaction between mental pain intensity and tolerance for mental pain was significant $(\beta=-0.007, S E=0.001, p<0.001$; see Table 2). The model predicted $36.1 \%$ of the variance in suicidal ideation and the interaction exclusively predicted $0.5 \%$ of the variance. Mental pain and all levels of tolerance for mental pain (low, average, and high) were significantly associated with suicidal ideation. Inspection of the source of the significant interaction is reported in Fig. 1 and indicates that

\footnotetext{
1 Rates of moderate to severe psychological distress were calculated with normative scores of the measures used to assess peritraumatic distress, anxiety, and depression. Specifically, the following cut-off scores were employed: CPDI, 28-51 = moderate distress, $\geq 52=$ severe distress (Qiu et al., 2020); GAD-7, 10-14=moderate anxiety, 15-21=severe anxiety (Spitzer et al., 2006); PHQ-9, 10-14=moderate depression, 15-19= moderately severe depression, 20-27 severe depression (Spitzer et al., 1999). Rates of suicidal ideation were reported based on responses to item 9 of the PHQ- 9 concerning suicidal risk (i.e., $0=$ not at all, $1=$ several days, $2=$ more than half of the days, and $3=$ nearly every day).
} 


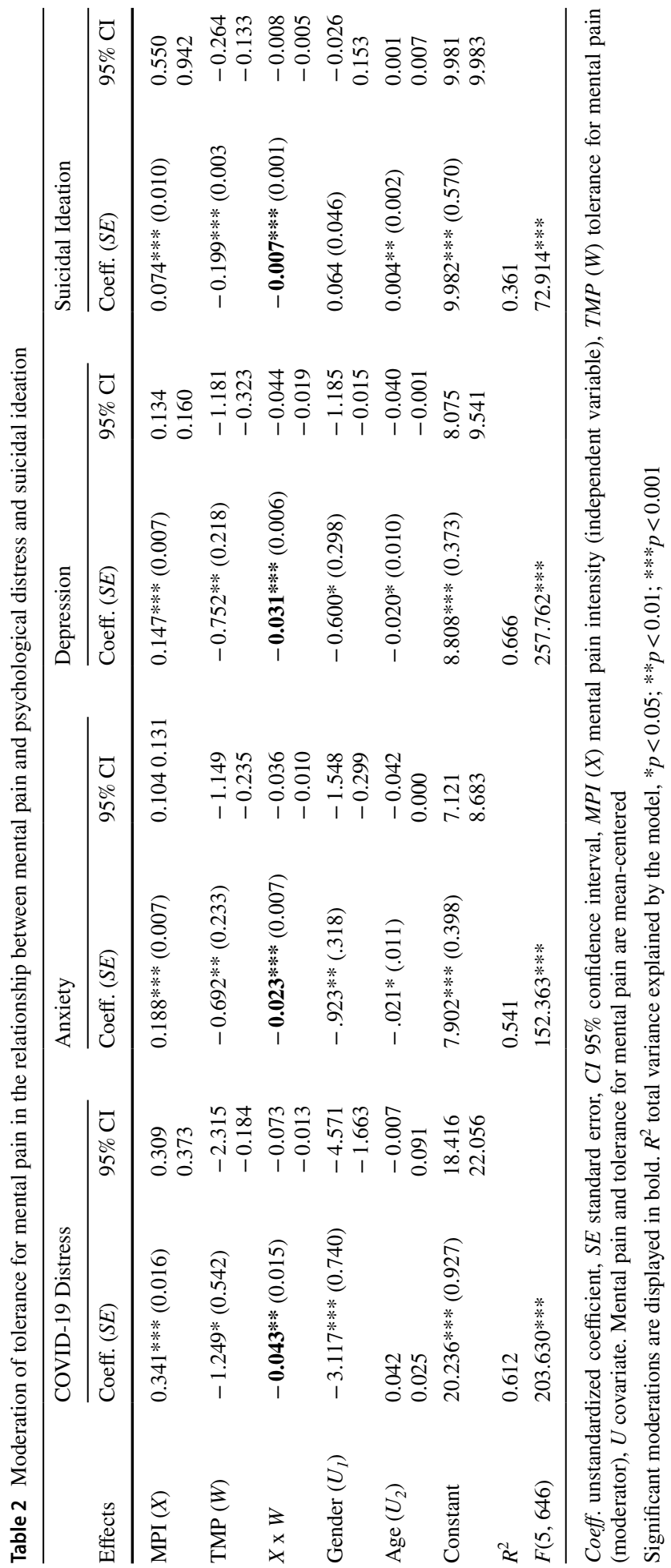



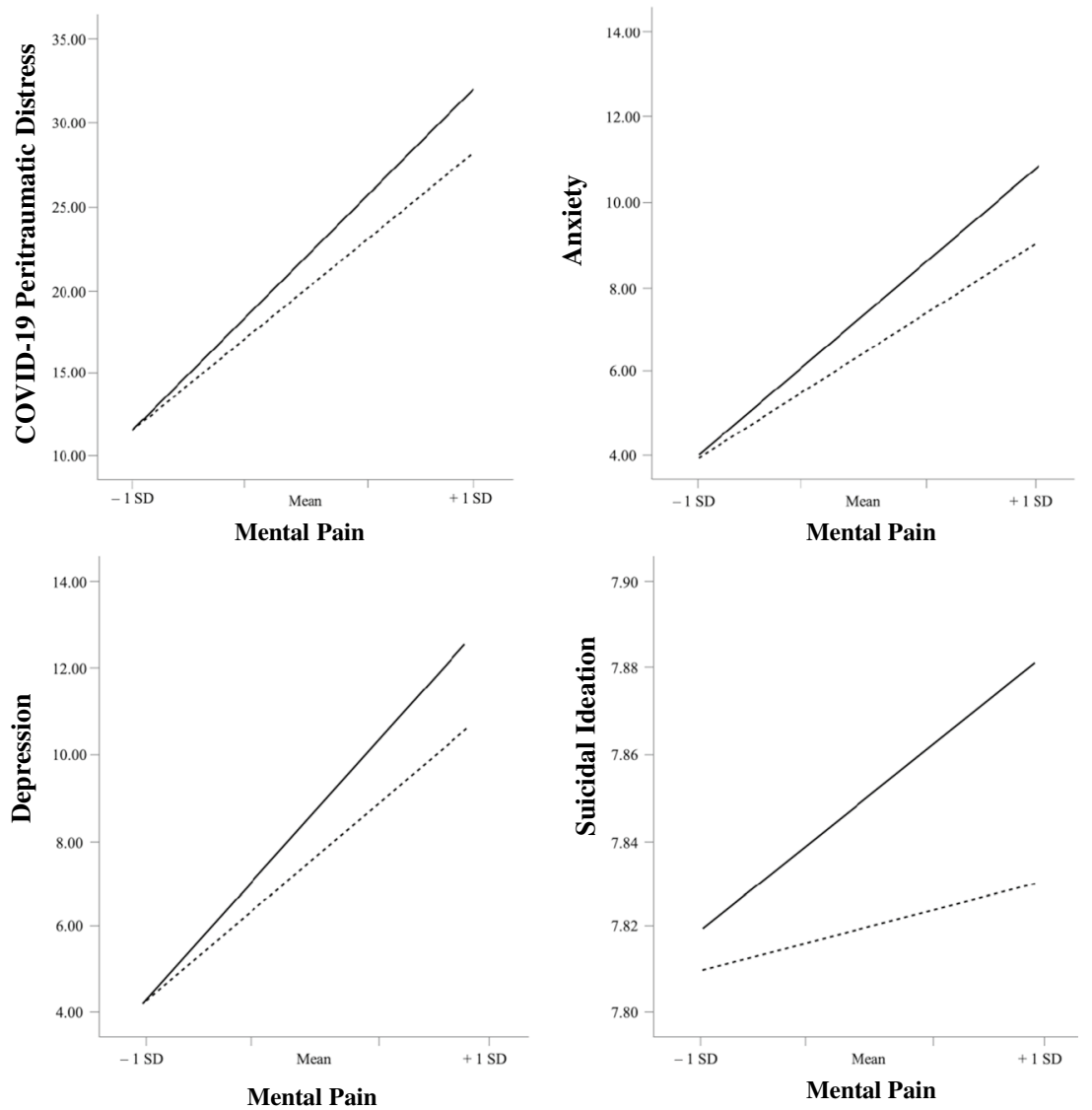

Tolerance for Mental Pain

$-1 \mathrm{SD}$

Fig. 1 The buffering effect of tolerance for mental pain on the link between mental pain and psychological distress and suicidal ideation. $S D$ standard deviation

higher tolerance for mental pain significantly weakened the positive link of mental pain intensity to suicidal ideation.

\section{Discussion}

The current study explored whether the intensity of mental pain is a risk factor for psychological distress and suicidal ideation during the COVID-19 pandemic and lockdown. As predicted, findings indicated that mental pain intensity was associated with heightened levels of psychological distress and suicidal ideation. These results are consistent with prior 
findings highlighting the role of mental pain intensity as a well-established predictor of suicidal risk (Levinger et al., 2015; Segal-Engelchin et al., 2015; Shelef et al., 2015) and its relation to various psychiatric disorders, such as depression and anxiety (e.g., Orbach et al., 2004). Moreover, our results expand previous research, by revealing a positive association between mental pain intensity and COVID-19-related psychological distress, further supporting the conceptualization of mental pain intensity as a transdiagnostic construct related to a wide array of psychiatric conditions (Orbach et al., 2003; Tossani, 2013). Mental pain in the context of the pandemic could be viewed as a broader representation of the intrasubjective suffering and helplessness experienced by the general population (Lifshin et al., 2020).

As a second aim, we examined the role of tolerance for mental pain, a psychosocial resource, in protecting people from psychological distress and suicidal ideation during the pandemic and in buffering the adverse mental health repercussions of mental pain intensity. Results indicated that tolerance for mental pain was a powerful protective factor, as it was significantly associated with lower levels of psychological distress and suicidal ideation during the pandemic. Moreover, higher levels of tolerance for mental pain significantly weakened the link of mental pain intensity to psychological distress and suicidal ideation. Suicide prevention measures in the context of a pandemic should be a key priority (Niederkrotenthaler et al., 2020; Moutier, 2021). This study empirically highlighted tolerance for mental pain as a resource that may be of particular importance (Gunnell et al., 2020). Being proactive in dealing with psychological suffering, increasing the ability to put the pain aside, and making efforts to keep it from overwhelmingly interfering with daily activities can be an invaluable psychological resource, especially during the current pandemic.

The current findings fit with previous research showing that higher tolerance for mental pain is predictive of less depressive symptomatology and reduced suicidal ideation (Landi et al., 2020a, b). Findings are also consistent with a recent study conducted on a US national sample of 10,368 adults during the COVID-19 pandemic in March 2020, in which depressive symptomatology, previously associated with mental pain, was shown to be a significant predictor of suicidality, while psychological resources, previously associated with mental pain tolerance, functioned as a protective factor (Fitzpatrick et al., 2020). Results are also aligned with another study conducted during the first lockdown in a sample of 1020 Spanish in which increased levels of fear of COVID-19, and lower interpersonal skills and resources - such as self-efficacy, social support, emotional self-regulation, etc.-were related to higher suicidal ideation (Soto-Sanz et al., 2020). Finally, the findings of this study are also in line with the broader literature highlighting positive psychological resources associated with resilience as important protective factors for adaptive psychological responses to the COVID-19 pandemic (Pakenham et al., 2020; Satici et al., 2020).

The results of the current study have numerous clinical implications. Because of the negative mental health impact of mental pain intensity during the COVID-19 lockdown and the adverse repercussions of previous epidemics on mental health and suicide (Zortea et al., 2020), it is important that public health interventions will be developed to bolster tolerance for psychological suffering during and after the COVID-19 outbreak. A recent study has further highlighted mental pain tolerance as associated with better psychotherapeutic outcomes, underlying the importance of implementing psychotherapeutic interventions during the current pandemic and reinforcing tolerance for mental pain within the interventions (Grossman-Giron et al., 2020).

Findings from the present study should be interpreted in the context of the following limitations. First, the online survey methodology and the exclusive reliance on self-report 
measures elevate the possibility of common-method variance. Second, the current crosssectional design limits inferences regarding the direction of causality of the link of mental pain intensity and tolerance to psychological distress and suicidal ideation. Longitudinal studies are needed to investigate causal links among these variables over time. Third, the fact that our convenience sample was comprised mostly of female participants compromises the generalizability of our findings to male participants. Fourth, we measured suicidal ideation with a single item. Future studies should use multi-item measures of suicidal ideation. Nevertheless, this study is the first to elucidate mental pain-suicidality risk and the protective role of tolerance for mental pain during the current COVID-19 pandemic.

\section{Conclusion}

Because of the alarming effects of the pandemic and lockdown on mental health and suicide (Bueno-Notivol et al., 2020; Dubé et al., 2021; Kan et al., 2021; Xiong et al., 2020), the current study underlines the protective role of tolerance for mental pain in reducing psychological distress and suicidal ideation during the pandemic. Evidence-based public health interventions focused on tolerance for mental pain during a pandemic are needed in order to effectively reduce suicide risk and promote resilience among the general population worldwide.

\section{Declarations}

Ethics Approval and Consent to Participate All procedures followed were in accordance with the ethical standards of a university institutional human research ethics committee and with the Helsinki Declaration of 1975 , as revised in 2000. Informed consent was obtained from all participants for being included in the study.

Conflict of Interest The authors declare no conflict of interest.

\section{References}

Ammerman, B. A., Burke, T. A., Jacobucci, R., \& McClure, K. (2021). Preliminary investigation of the association between COVID-19 and suicidal thoughts and behaviors in the US. Journal of Psychiatric Research, 134, 32-38. https://doi.org/10.1016/j.jpsychires.2020.12.037

Bakioğlu, F., Korkmaz, O., \& Ercan, H. (2020). Fear of COVID-19 and positivity: Mediating role of intolerance of uncertainty, depression, anxiety, and stress. International Journal of Mental Health and Addiction, 1-14. Advance online publication. https://doi.org/10.1007/s11469-020-00331-y

Becker, G., Orbach, I., Mikulincer, M., Iohan, M., Gilboa-Schechtman, E., \& Grossman-Giron, A. (2019). Reexamining the mental pain-suicidality link in adolescence: The role of tolerance for mental pain. Suicide and Life Threating Behavior, 49(4), 1072-1084. https://doi.org/10.1111/sltb.12506

Besser, A., Flett, G. L., Nepon, T., \& Zeigler-Hill, V. (2020). Personality, cognition, and adaptability to the COVID-19 Pandemic: Associations with loneliness, distress, and positive and negative mood states. International Journal of Mental Health and Addiction, 1-25. Advance online publication. https://doi. org/10.1007/s11469-020-00421-x

Bolger, E. (1999). Grounded theory analysis of emotional pain. Psychotherapy Research, 9(3), 342-362. https://doi.org/10.1080/10503309912331332801

Brooks, S. K., Webster, R. K., Smith, L. E., Woodland, L., Wessely, S., Greenberg, N., \& Rubin, G. J. (2020). The psychological impact of quarantine and how to reduce it: Rapid review of the evidence. The Lancet, 395(10227), 912-920. https://doi.org/10.1016/s0140-6736(20)30460-8

Bueno-Notivol, J., Gracia-García, P., Olaya, B., Lasheras, I., López-Antón, R., \& Santabárbara, J. (2020). Prevalence of depression during the COVID-19 outbreak: A meta-analysis of community-based 
studies. International Journal of Clinical and Health Psychology. https://doi.org/10.1016/j.ijchp.2020. 07.007

Curran, P. J., West, S. G., \& Finch, J. F. (1996). The robustness of test statistics to nonnormality and specification error in confirmatory factor analysis. Psychological Methods, 1(1), 16-29. https://doi.org/10. 1037/1082-989X.1.1.16

Daly, M., \& Robinson, E. (2021). Psychological distress and adaptation to the COVID-19 crisis in the United States. Journal of Psychiatric Research, 136, 603-609. https://doi.org/10.1016/j.jpsychires. 2020.10.035

Dubé, J. P., Smith, M. M., Sherry, S. B., Hewitt, P. L., \& Stewart, S. H. (2021). Suicide behaviors during the COVID-19 pandemic: A meta-analysis of 54 studies. Psychiatry Research, 301, 113998. https://doi. org/10.1016/j.psychres.2021.113998

Fitzpatrick, K. M., Harris, C., \& Drawve, G. (2020). How bad is it? Suicidality in the middle of the COVID19 pandemic. Suicide and Life-Threatening Behavior. https://doi.org/10.1111/sltb.12655

Grossman-Giron, A., Becker, G., Kivity, Y., Shalev, S., \& Tzur Bitan, D. (2020). Mental pain intensity and tolerance as predictors of psychotherapy process and outcome. Journal of Clinical Psychology. https:// doi.org/10.1002/jclp. 23085

Gunnell, D., Appleby, L., Arensman, E., Hawton, K., John, A., Kapur, N., ... \& Chan, L. F. (2020). Suicide risk and prevention during the COVID-19 pandemic. The Lancet Psychiatry 7(6), 468-471. https://doi. org/10.1016/S2215-0366(20)30171-1

Hayes, A. F. (2018). Introduction to mediation, moderation, and conditional process analysis: A regressionbased approach. The Guildford Press.

Iob, E., Steptoe, A., \& Fancourt, D. (2020). Abuse, self-harm and suicidal ideation in the UK during the COVID19 pandemic. The British Journal of Psychiatry, 217(4), 543-546. https://doi.org/10.1192/bjp.2020.130

Kan, F. P., Raoofi, S., Rafiei, S., Khani, S., Hosseinifard, H., Tajik, F., ... \& Ghashghaee, A. (2021). A systematic review of the prevalence of anxiety among the general population during the COVID-19 pandemic. Journal of Affective Disorders, 293, 391-398https://doi.org/10.1016/j.jad.2021.06.073

Kroenke, K., \& Spitzer, R. L. (2010). Instruction manual: Instructions for patient health questionnaire (PHQ) and GAD-7 measures. www.phqscreeners.com. Accessed 18 Mar 2019.

Landi, G., Furlani, A., Boccolini, G., Mikulincer, M., Grandi, S., \& Tossani, E. (2020a). Tolerance for Mental Pain Scale (TMPS): Italian validation and evaluation of its protective role in depression and suicidal ideation. Psychiatry Research, 291, 113263. https://doi.org/10.1016/j.psychres.2020.113263

Landi, G., Pakenham, K. I., Boccolini, G., Grandi, S., \& Tossani, E. (2020). Health anxiety and mental health outcome during COVID-19 lockdown in Italy: The mediating and moderating roles of psychological flexibility. Frontiers in Psychology, 11, 2195. https://doi.org/10.3389/fpsyg.2020.02195

Leaune, E., Samuel, M., Oh, H., Poulet, E., \& Brunelin, J. (2020). Suicidal behaviors and ideation during emerging viral disease outbreaks before the COVID-19 pandemic: A systematic rapid review. Preventive Medicine, 141, 106264. https://doi.org/10.1016/j.ypmed.2020.106264

Levinger, S., Somer, E., \& Holden, R. R. (2015). The importance of mental pain and physical dissociation in youth suicidality. Journal of Trauma \& Dissociation, 16(3), 322-339. https://doi.org/10.1080/15299 732.2014 .989644

Lifshin, U., Mikulincer, M., \& Kretchner, M. (2020). Motivated helplessness in the context of the Covid-19 Pandemic: Evidence for a curvilinear relationship between perceived ability to avoid the virus and anxiety. Journal of Social and Clinical Psychology, 39(6), 479-497. https://doi.org/10.1521/jscp.2020.39.6.479

Mamun, M. A., Chandrima, R. M., \& Griffiths, M. D. (2020). Mother and son suicide pact due to COVID-19-related online learning issues in Bangladesh: An unusual case report. International Journal of Mental Health and Addiction, 1-4. Advance online publication. https://doi.org/10.1007/ s11469-020-00362-5

Meerwijk, E. L., \& Weiss, S. J. (2018). Tolerance for psychological pain and capability for suicide: Contributions to suicidal ideation and behavior. Psychiatry Research, 262, 203-208. https://doi.org/10. 1016/j.psychres.2018.02.005

Meerwijk, E. L., Mikulincer, M., \& Weiss, S. J. (2019). Psychometric evaluation of the tolerance for mental pain scale in United States adults. Psychiatry Research, 273, 746-752. https://doi.org/10.1016/j.psych res.2019.01.101

Montemarano, V., Troister, T., Lambert, C. E., \& Holden, R. R. (2018). A four-year longitudinal study examining psychache and suicide ideation in elevated-risk undergraduates: A test of Shneidman's model of suicidal behavior. Journal of Clinical Psychology, 74(10), 1820-1832. https://doi.org/10. $1002 /$ jclp. 22639

Moutier, C. (2021). Suicide prevention in the COVID-19 era: Transforming threat into opportunity. JAMA Psychiatry, 78(4), 433-438. https://doi.org/10.1001/jamapsychiatry.2020.3746 
Niederkrotenthaler, T., Braun, M., Pirkis, J., Till, B., Stack, S., Sinyor, M., ... \& Scherr, S. (2020). Association between suicide reporting in the media and suicide: A systematic review and meta-analysis. $B M J, 368$. https://doi.org/10.1136/bmj.m575

Niederkrotenthaler, T., Gunnell, D., Arensman, E., Pirkis, J., Appleby, L., Hawton, K., ... \& Platt, S. (2020). Suicide research, prevention, and COVID-19. Crisis (2020), 41(5), 321-330 https://doi.org/10.1027/ 0227-5910/a000731

Orbach, I., Gilboa-Schechtman, E., Johan, M., \& Mikulincer, M. (2004). Tolerance for mental pain scale. Bar-Ilan University.

Orbach, I., Mikulincer, M., Gilboa-Schechtman, E., \& Sirota, P. (2003). Mental pain and its relationship to suicidality and life meaning. Suicide and Life-Threatening Behavior, 33(3), 231-241. https://doi.org/ 10.1521/suli.33.3.219.23219

Pakenham, K. I., Landi, G., Boccolini, G., Furlani, A., Grandi, S., \& Tossani, E. (2020). The moderating roles of psychological flexibility and inflexibility on the mental health impacts of COVID-19 pandemic and lockdown in Italy. Journal of Contextual Behavioral Science, 17, 109-118. https://doi.org/ 10.1016/j.jcbs.2020.07.003

Qiu, J., Shen, B., Zhao, M., Wang, Z., Xie, B., \& Xu, Y. (2020). A nationwide survey of psychological distress among Chinese people in the COVID-19 epidemic: Implications and policy recommendations. General Psychiatry, 33, e100213. https://doi.org/10.1136/gpsych-2020-100213

Satici, S. A., Kayis, A. R., Satici, B., Griffiths, M. D., \& Can, G. (2020). Resilience, hope, and subjective happiness among the Turkish population: Fear of COVID-19 as a Mediator. International Journal of Mental Health and Addiction, 1-16. Advance online publication. https://doi.org/10.1007/ s11469-020-00443-5

Segal-Engelchin, D., Kfir-Levin, N., Neustaedter, S. B., \& Mirsky, J. (2015). Mental pain among female suicide attempt survivors in Israel: An exploratory qualitative study. International Journal of Mental Health and Addiction, 13(4), 423-434. https://doi.org/10.1007/s11469-015-9545-2

Shelef, L., Fruchter, E., Hassidim, A., \& Zalsman, G. (2015). Emotional regulation of mental pain as moderator of suicidal ideation in military settings. European Psychiatry, 30(6), 765-769. https://doi.org/ 10.1016/j.eurpsy.2014.12.004

Shneidman, E. S. (1993). Suicide as a psychache. Journal of Nervous and Mental Disease, 181, 147-149. https://doi.org/10.1097/00005053-199303000-00001

Simon, G. E., Rutter, C. M., Peterson, D., Oliver, M., Whiteside, U., Operskalski, B., \& Ludman, E. J. (2013). Does response on the PHQ-9 depression questionnaire predict subsequent suicide attempt or suicide death? Psychiatric Services, 64, 1195-1202. https://doi.org/10.1176/appi.ps.201200587

Soto-Sanz, V., Falcó, R., \& Piqueras, J. A. (2020). Socio-emotional strengths against psychopathology and suicidal ideation in fear of Covid-19. Preprint, available at Research Square. https://doi.org/10.21203/rs.3.rs-73382/v1

Spitzer, R. L., Kroenke, K., \& Williams, J. B. (1999). Validation and utility of a self-report version of PRIME-MD: The PHQ primary care study. JAMA, 282(18), 1737-1744. https://doi.org/10.1001/jama. 282.18.1737

Spitzer, R. L., Kroenke, K., Williams, J. B., \& Löwe, B. (2006). A brief measure for assessing generalized anxiety disorder: The GAD-7. Archives of Internal Medicine, 166(10), 1092-1097. https://doi.org/10. 1001/archinte.166.10.1092

Tossani, E. (2013). The concept of mental pain. Psychotherapy and Psychosomatics, 82(2), 67-73. https:// doi.org/10.1159/000343003

Tossani, E., Ricci Garotti, M. G., Mikulincer, M., Giovagnoli, S., Calzolari, G., Landi, G., \& Grandi, S. (2021). Psychometric evaluation of the Italian version of Orbach \& Mikulincer mental pain scale in a non-clinical sample. Current Psychology, 40(4), 1903-1910. https://doi.org/10.1007/ s12144-019-0128-4

Verrocchio, M. C., Carrozzino, D., Marchetti, D., Andreasson, K., Fulcheri, M., \& Bech, P. (2016). Mental pain and suicide: A systematic review of the literature. Frontiers in Psychiatry, 7, 108. https://doi.org/ 10.3389/fpsyt.2016.00108

Xiong, J., Lipsitz, O., Nasri, F., Lui, L. M., Gill, H., Phan, L., ... \& McIntyre, R. S. (2020). Impact of COVID-19 pandemic on mental health in the general population: A systematic review. Journal of Affective Disorders, 277(1), 55-64. https://doi.org/10.1016/j.jad.2020.08.001

Zortea, T. C., Brenna, C. T., Joyce, M., McClelland, H., Tippett, M., Tran, M. M., ... \& Links, P. (2020). The impact of infectious disease-related public health emergencies on suicide, suicidal behavior, and suicidal thoughts. Crisis, 16, 1-14. https://doi.org/10.1027/0227-5910/a000753

Publisher's Note Springer Nature remains neutral with regard to jurisdictional claims in published maps and institutional affiliations. 